\title{
Oral Health, Nutritional Choices, and Dental Fear and Anxiety
}

\author{
Jennifer R. Beaudette ${ }^{1}$, Peter C. Fritz ${ }^{1,2}$, Philip J. Sullivan ${ }^{1}$ and Wendy E. Ward ${ }^{1, *}$ \\ 1 Department of Kinesiology, Brock University, St. Catharines, ON L2S 3A1, Canada; \\ jb10im@brocku.ca (J.R.B.); drpeterfritz@me.com (P.C.F.); psullivan@brocku.ca (P.J.S.) \\ 2 Reconstructive Periodontics and Implant Surgery Clinic, Fonthill, ON L0S 1E5, Canada \\ * Correspondence: wward@brocku.ca; Tel.: +1-905-688-5550 (ext. 3024)
}

Academic Editor: Nick Girdler

Received: 4 November 2016; Accepted: 16 January 2017; Published: 21 January 2017

\begin{abstract}
Oral health is an integral part of overall health. Poor oral health can lead to an increased risk of chronic diseases including diabetes mellitus, cardiovascular disease, and some types of cancer. The etiology of these diseases could be linked to the individual's inability to eat a healthy diet when their dentition is compromised. While periodontal or implant surgery may be necessary to reconstruct tissue around natural teeth or replace missing teeth, respectively, some individuals avoid such interventions because of their associated fear and anxiety. Thus, while the relationship between poor oral health, compromised nutritional choices and fear and anxiety regarding periodontal procedures is not entirely new, this review provides an up-to-date summary of literature addressing aspects of this complex relationship. This review also identifies potential strategies for clinicians to help their patients overcome their fear and anxiety associated with dental treatment, and allow them to seek the care they need.
\end{abstract}

Keywords: anxiety; fear; nutrition; oral health; periodontal

\section{Introduction}

This review will identify what is known about the complex relationship among poor oral health, compromised nutritional choices, and fear and anxiety regarding periodontal procedures. Specifically, tooth loss and how it affects food and/or nutrient intake will be discussed as well as how dental fear and anxiety can lead patients to avoid dental treatment, thereby potentially further compromising their oral health through an inability to consume a healthful diet. Some strategies to help patients manage their dental anxiety are briefly discussed. The articles cited in this literature review were found by searching a number of online databases including Medline, Web of Science Complete, and Academic Search Complete. Examples of some of the search terms used in combination are: oral health, nutrition, nutrient intake, dental status, dental fear, anxiety and periodontal.

Nutrition and oral health are inextricably linked. Poor oral health can affect an individual's ability to eat certain nutritious foods while poor nutrition can increase an individual's risk of poor oral health including periodontal disease and tooth loss. Periodontal disease has been linked to diabetes mellitus, cardiovascular disease, and some types of cancer [1-3]. Tooth loss, which may or may not be related to periodontal disease, has been associated with an increased risk of a number of chronic diseases, including coronary heart disease and chronic kidney disease [4,5]. It may also be associated with poor nutrient intake. Epidemiological evidence suggests a diet high in fruits, vegetables, and whole grains can decrease the risk of coronary heart disease [6]. Moreover a diet high in vitamin C, which is found in many of the fruits and vegetables that individuals with poor oral health might find difficult to eat, is protective against some types of cancers, including of the mouth [6]. Thus, poor nutrient intake that originates from compromised dental status may result in a higher risk of chronic disease. 
Replacing and maintaining natural teeth improves masticatory ability, allowing individuals to consume a varied and nutritious diet, and is a strategy to end the cycle that can set an individual on a trajectory for chronic disease development (Figure 1). However, while reconstructive periodontal surgery and/or implant placement can avoid limiting food choices due to poor dentition, many people refuse or delay these procedures because of fear and anxiety (Figure 1). Identifying and implementing strategies to reduce fear and anxiety among patients could end the cycle by encouraging more patients to seek necessary treatment; leading to greater oral and overall health.

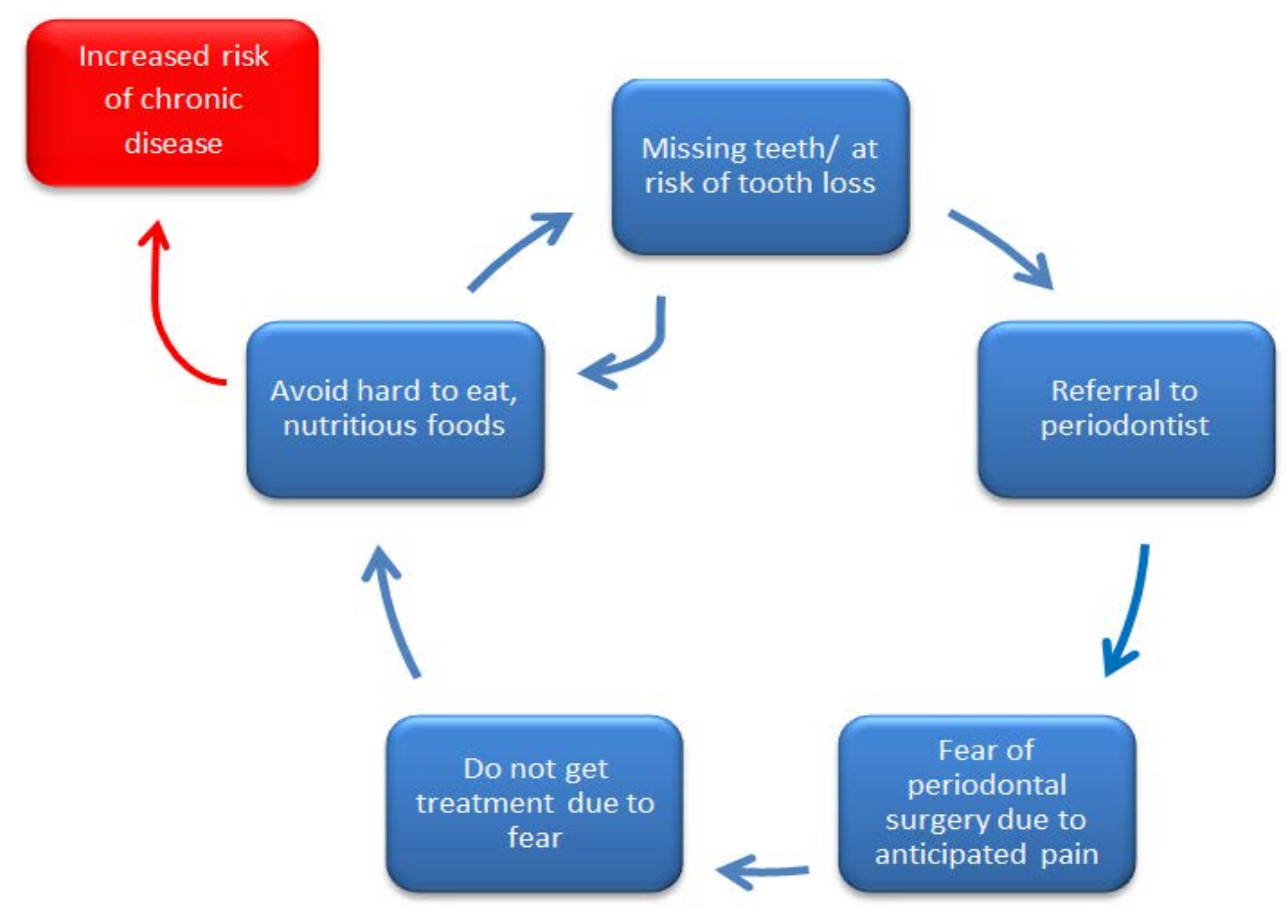

Figure 1. It is hypothesized that individuals can find themselves cycling between poor oral health (missing teeth or at risk of tooth loss) and poor nutrition (avoidance of hard to eat, nutritious foods) because they fear the necessary dental treatment that can break the cycle. Remaining in the cycle between poor oral health and poor nutrition may put an individual at an increased risk of developing chronic disease.

\section{Tooth Loss}

The Oral Health Module of the Canadian Health Measures Survey revealed that $6.4 \%$ of Canadian adults (aged 20-79) are edentulous [7]. When broken down by age groups, the highest rate of edentulism was among older adults (aged $60-79$ ) at $21.7 \%$ compared to the $40-59$ year old age group with a rate of $4.4 \%$. A full complement of teeth is considered to be 28 teeth, although an individual can have up to 32 teeth if there is no tooth loss and all four third molars are present. The average number of teeth that Canadian adults have is 24.53 teeth. Of the $93.6 \%$ of dentate Canadian adults, $42.3 \%$ have all 28 teeth, 36.7\% have between 28 and 21 teeth, and 14.6\% have fewer than 21 teeth [7]. A total of $57.7 \%$ of Canadian adults are missing one or more teeth and an impaired dentition can lead to negative health outcomes [7].

Data from the Global Burden of Disease study showed that in 2010, 3.9 billion people were affected by oral health conditions [8]. Untreated dental caries of permanent teeth were the most prevalent of these conditions, affecting $35 \%$ of the global population. Severe periodontitis had a global prevalence of $11 \%$ and severe tooth loss ( $<9$ remaining teeth) had a global prevalence of $2 \%$ [8]. In the US, the prevalence of periodontitis was assessed by the National Health and Nutrition Examination Survey (NHANES) [9]. Beginning in 2009, a full-mouth periodontal examination was done as opposed to the partial-mouth examination that has previously been used. NHANES 2011-2012 found that 
$44.7 \%$ of adults aged 30 years and older had periodontitis, which is similar to the $47.2 \%$ found in the 2009-2010 survey [9]. The prevalence of periodontitis was higher among those living below the federal poverty line, those who had less than a high school education, and those who were current smokers [9]. Using disability adjusted life years (DALYs), it was found that oral health conditions contributed to 224 years of healthy living lost per 100,000 people [8].

\section{Dentition and Food Choices}

The association between periodontal disease and/or tooth loss and increased risk of chronic disease can, at least partially, be related to diet (Table 1). When dietary intakes were evaluated using 24-h recall questionnaires or food frequency questionnaires, it was found that individuals who are missing teeth tend to consume fewer servings of fruits and vegetables, less fiber, and more cholesterol [10-12]. This may be because missing teeth can limit an individual's food choices. It can also impact the method they choose to cook their foods. Foods that have a more healthful nutrient profile are often more difficult to masticate, particularly for older adults. Examples include fruits, raw vegetables, and meats.

A study of Finnish adults aged 30 years and older included 7190 dental patients and assessed dietary intakes using a 24-h dietary intake recall questionnaire [13]. These patients were divided based on their dental status of dentate or edentulous. The dentate group was further divided into those who had removable prosthetics and those who did not. The edentulous group was divided into those who had both an upper and lower denture, which was considered adequate rehabilitation and those who had only one denture (either upper or lower) or no dentures, which was considered inadequate rehabilitation. The dentate population ate more fruits and vegetables, including root vegetables, than the edentulous population. Among the dentate group, having a higher number of natural teeth increased the probability of the participant eating fruits, vegetables (including root vegetables), and meat. Analysis of the edentulous group showed that having adequate rehabilitation was positively associated with eating more fruits, vegetables, and root vegetables. No difference was found between the adequacy of dental rehabilitation and the likelihood that they consumed an easy to chew food such as porridge within either the dentate or edentulous group [13]. It can therefore be inferred that individuals who lack adequate rehabilitation for their missing teeth avoid foods that are harder to chew such as fruits and raw vegetables. The impaired ability to eat fruits and raw vegetables could lead to an increased risk of chronic disease due to lower intakes of polyphenols that may act as antioxidants. Although it varies by vegetable and cooking method, the polyphenol content of some cooked vegetables (i.e., carrots, broccoli) is lower than the raw form [14]. Thus, the antioxidant capacity of vegetables can also be altered in the cooking process changing their ability to scavenge free radicals [14]. Some vegetables are best consumed raw to get the full antioxidant benefit, but consuming these foods can be difficult when the dentition is compromised.

The relationship between dentition status and food choice was examined for adults aged 65 years and older who participated in the British National Diet and Nutrition Survey [15]. An oral examination was performed for each participant. The number of contact points between opposing teeth (top and bottom) were recorded for dentate individuals (anyone with some remaining natural teeth), as there is evidence to suggest this affects one's ability to chew effectively [16]. All the edentulous participants had dentures. Data on food choice was gathered by giving participants a list of sixteen food items that ranged in masticatory difficulty. Examples include bread, carrots and steak. Participants were asked to indicate the level of difficulty they would experience when eating the specific food item. Specifically, they were asked whether they could eat it easily, with some difficulty, or not at all. Within the dentate group, $28 \%$ of participants reported they would have difficulty eating apples or would not be able to eat apples at all. That number increased to $50 \%$ within the edentulous group. The differences between the dentate compared to the edentulous groups' anticipated ability to eat tomatoes, raw carrots, apples, and nuts were significant, while the anticipated ability to eat foods such as bread, toast, cheese, roasted potatoes, cooked greens, and chocolate did not differ significantly among groups. It was also found 
that number of natural teeth remaining affected the anticipated ability to eat certain foods within the dentate group. Of the participants with one to 10 teeth, $45 \%$ reported that they would have difficulty eating or would not be able to eat apples whereas of the participants with 21 or more teeth, only $12 \%$ reported anticipated difficulty or inability to eat apples. A total of $26 \%$ of participants with 11-20 teeth reported they would have difficulty or be unable to eat apples [15]. Apple consumption is associated with health benefits including decreased risk of obesity and cardiovascular disease $[17,18]$. The decreased risk of cardiovascular disease is often attributed to their high fiber and polyphenol content [18]. Ability to eat other fruits and vegetables of similar texture can be inferred by ability to eat apples.

A longitudinal survey of 30,000 participants investigated dietary intake of those who lost five or more teeth compared to those who lost no teeth during a four-year follow up [10]. A diet questionnaire was used to gather intake data about 131 foods and supplements [10]. The questionnaire revealed that individuals who lost teeth significantly reduced their intake of apples and pears in the follow-up period. Similar to other studies, the intake of pears, apples, and carrots increased as the number of teeth increased [10]. Knowing that dental status affects an individual's ability to eat certain nutritious foods such as raw vegetables or various fruits and even some meats, there is concern that people with inadequate dentition can be at risk for nutritional deficiencies. They can also be more likely to consume foods that are softer in texture, but often tend to be higher in saturated fat and cholesterol. For example, edentulous participants in the longitudinal study consumed more saturated fat, cholesterol, and had a higher caloric intake than participants with 25 or more teeth [10]. One study has shown that $86 \%$ of individuals with 20 or more teeth allowed them to consume apples, carrots and steaks (cooked as "well-done") with ease [15]. It has also been suggested that a better way to assess chewing ability is the number of posterior occluding pairs (POPs) that an individual has. POPs are the number of molars or premolars where the opposing mandibular and maxillary teeth are present [19]. Individuals with five pairs of POPs, out of a possible eight, were able to consume a wider variety of nutritious foods, i.e., fruit and raw vegetables, which resulted in a higher quality diet based on the Healthy Eating Index (HEI) [11]. Individuals with fewer than five POPs were more likely to avoid certain foods and eat a less varied and nutritious diet.

While it has been shown that missing natural teeth limits food choice because people avoid foods that they find difficult to consume, it has not been well established if these limitations lead to specific nutrient deficiencies. The evidence in support of this, however, is growing. In a study of male health professionals that studied food and nutrient intake via a questionnaire, edentulous participants consumed significantly fewer vegetables and dietary fiber than their counterparts with 25 or more teeth [10]. The edentulous participants' intake of carotenes and crude fiber was also significantly lower, while total caloric intake, cholesterol, and saturated fat intake were significantly higher than those with 25 or more teeth. This highlights the fact that diet quality differs between these groups. The results of the questionnaire showed that there was no significant difference between groups for total fruit or vitamin C intake [10]. It is possible that because of their higher energy intake, edentulous individuals are getting most of the nutrients they need, but they are getting them from less healthful foods.

To gain a more accurate sense of nutrients consumed, serum markers of nutrient status can be measured and generally provide more accurate information than a dietary record. A study of participants aged 65 and older included a four-day food diary in addition to blood and urine analyses as a surrogate measure of nutrient intake [20]. The sample consisted of 407 dentate individuals and 346 edentulous participants (endentulous participants all had dentures). Analysis of the food diaries showed that edentulous participants consumed less protein, intrinsic sugars, milk sugars, fiber, calcium, non-heme iron, riboflavin, thiamin, niacin, pantothenic acid, vitamin E, and vitamin C. Blood and urine analyses revealed that only plasma ascorbate and plasma retinol were statistically different between the dentate and edentulous participants. When the food diaries for the dentate individuals were analyzed based on number of teeth, those with more teeth reported higher intake of protein, fat, and carbohydrate as well as fiber, intrinsic sugar, milk sugar, calcium, non-heme iron, pantothenic acid, 
vitamin C, and vitamin E. Intake of these nutrients was significantly associated with number of POPs, as were vitamin A, thiamin, riboflavin, and niacin. Only plasma ascorbate was significantly associated with the number of teeth remaining; those with more teeth had higher plasma ascorbate levels. Plasma retinol was lower in the edentulous group than the dentate group, but was not associated with number of teeth remaining or POPs [20]. Individuals who are missing teeth might not be at as high of a risk for deficiencies of specific nutrients as previously thought based on biochemical measures, but some nutrients, such as beta-carotene, were not measured.

Table 1. Summary of findings regarding dentition and food choices.

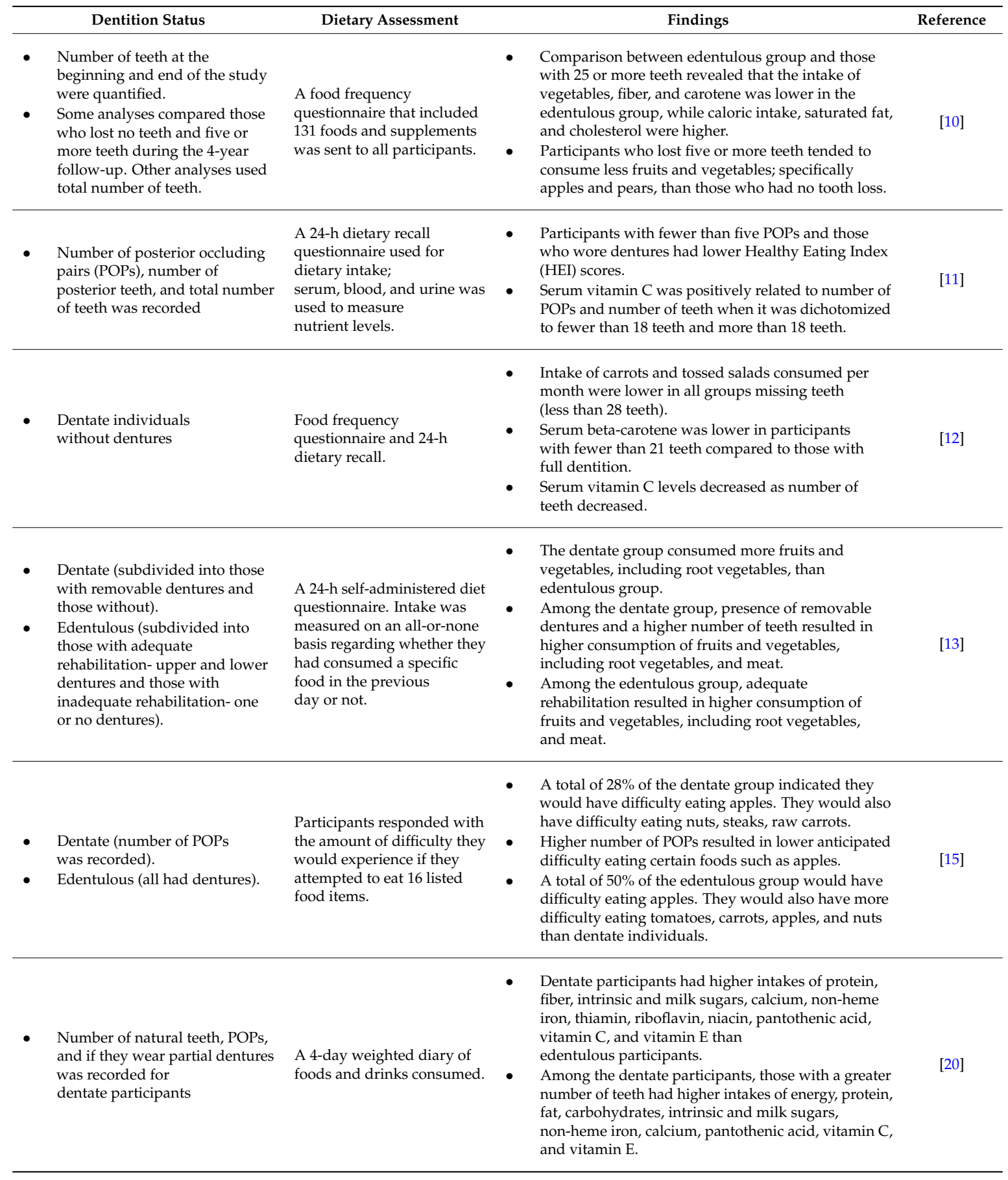

A similar study was conducted using data from the third National Health and Nutrition Examination Survey (NHANES III) in which dietary intake was collected using a 24-h recall 
method [11]. Serum levels of vitamin C, vitamin E, folate, and beta-carotene were selected as indicators of nutritional status. Carotenes, folacin, and ascorbic acid were significantly lower for people with one to four POPs than people with five to eight POPs. Vitamin A was significantly lower for edentulous participants and participants who wore full dentures, and dietary fiber was highest for those with five to eight POPs and lowest in the edentulous group. When serum levels of these nutrients were measured, it was found that only beta-carotene and vitamin $C$ were significantly associated with number of POPs. There was a positive relationship; a higher number of POPs was associated with a higher serum level of vitamin $C$ and beta-carotene. Serum folate was lower in denture wearers than it was in people with five to eight POPs [11]. In both aforementioned studies, vitamin C status was associated with number of teeth or POPs remaining $[11,20]$. One possible explanation for this is that many of the foods that are good sources of vitamin C - broccoli, brussels sprouts and carrots-must be cooked and softened to allow consumption by people with missing teeth. Depending on the method of cooking, this can decrease the amount of vitamin C available by up to $38 \%$ [21].

\section{Pervasiveness of Fear and Anxiety toward Dental Treatment and Dental Avoidance Behaviours}

Among Canadian adults, the prevalence of dental anxiety ranges from $4.4 \%$ to $16.4 \%$ [22]. The prevalence among Australian adults was similar with $16.1 \%$ of a random sample reporting high dental fear [23]. In both studies, females were more likely to report having high dental fear than males [22,23]. It is not well understood why females have higher dental fear. In a survey looking at attitudes toward dental fear, women were shown to fear pain more than men [24]. A study that aimed to understand gender differences in pain expectations before surgery and the memory of pain following surgery found that women had significantly greater anxiety than men before and after surgery. Interestingly, although women expressed greater anxiety, they expected less pain prior to the periodontal treatment than men [25]. The gender differences of reported anxiety or fear are often attributed to societal expectations for men to be tough, which could lead to them underreporting their true fear and anxiety toward dental treatment. One study sought to show that societal expectations played a role in the reporting of dental fear between men and women by changing the wording "fear" with "dread" [26]. They hypothesized that men would admit feeling dread toward various dental treatments because dread is more socially acceptable than fear. This was partially supported in that they did find men were more likely to express dread than fear, but it was not isolated to men as women were also more likely to express dread than fear [26]. In the Canadian sample, there were no age differences found, but in the Australian sample, those aged 40 to 64 years were more likely to report high fear of dental treatment $[22,23]$. The two studies grouped patients by age using different cut points; this could account for the different findings. For instance, the Canadian sample was divided into six age groups ( 18 to 24 years, 25 to 34 years, 35 to 44 years, 45 to 54 years, 55 to 64 years, 65 years and older) while the Australian sample was divided into seven age groups that included a broader range of ages (under 13 years, 13 to 17 years, 18 to 24 years, 25 to 39 years, 40 to 64 years, 65 to 79 years, 80 years and older).

High dental fear is a major reason why a patient may choose to miss and cancel appointments or to avoid dental appointments. When asked about why they had avoided the dentist in the past year, one study found that fear of dental treatment was the reason given by $7.8 \%$ of participants [22]. When patients were categorized as having a high level of dental fear versus those with low or no dental fear, those in the high dental fear group reported missing, cancelling, or avoiding the dentist significantly more than those in the low or no fear group [22]. Although fear is a major contributor to the reasons people avoid dental treatment it should be noted that it is not the only reason cited for not seeking dental treatment. Other common reasons people give for not seeking dental treatment include a lack of time, the cost of treatment, the feeling that treatment was not required, the concern about the pain associated with treatment, and a dislike of dentists [27,28].

Several studies have reported main factors that lead to avoiding or delaying dental treatment. In one study, reasons for avoiding the dentist in the past year included teeth had not been bothering 
them $(40.7 \%)$, cost $(30.7 \%)$, lack of time $(14.9 \%)$, fear of dental treatment $(7.8 \%)$, and other reasons $(5.9 \%)$ [22]. When young adults (mean age $23.2 \pm 4.0$ years) were surveyed regarding their dental care practices, $36 \%$ of respondents reported "lack of time" as a reason for irregular visits [28]. A total of $34.1 \%$ reported that they did not have regular attendance because they felt that treatment was not needed, while $16.6 \%$ reported that cost of treatment limited their attendance, and $13.1 \%$ reported that fear kept them from the dentist [28]. Among the participants, those who had regular dental attendance reported lower dental anxiety than those who had irregular attendance (20.9\% versus $79.1 \%$ ) [28]. A different study divided patients into high and low dental anxiety groups based on responses to the Index of Dental Anxiety and Fear (IDAF-4C) [27]. All of the patients were asked if they were currently avoiding the dentist and the reason behind their avoidance. Patients in both the high and low dental anxiety groups reported cost as the most common reason for avoidance $(72.5 \%$ versus $70.0 \%$, respectively). In the high dental anxiety group, the next most common reasons for avoidance included fear or anxiety (55.6\%), dislike of the dentist (41.2\%), and concern about the pain or having an unpleasant experience (41.1\%) [27]. In the low dental anxiety group, the second most common reason for avoidance following cost was lack of time (38.8\%) [27]. There are a number of factors that contribute to a patient's anxiety leading up to a dental visit, with fear of the pain associated with treatment as a primary cause, which can ultimately lead to avoidance of dental treatment.

Dental fear can lead to long-term avoidance of visiting a dentist. More patients who had not been to the dentist in the previous 10 years had more dental fear than those who had been to the dentist within the previous 12 months [23]. Avoiding treatment can reinforce the fear of dental treatment because it often results in patients seeking dental treatment only when the oral health condition has deteriorated to such a degree that intensive treatment is required. In a study where patients reported avoiding the dentist for an average of 7.5 years (ranged from 0 to 40 years), it was found that the number of years an individual had avoided the dentist was correlated with poorer oral health [29]. Fear of dental treatment can also be reinforced when patients with high dental anxiety only seek treatment on a problem-oriented basis because a delay in seeking treatment can lead to more complicated clinical situations that might have been prevented with earlier intervention [27].

\section{Impact of Fear and Anxiety on Periodontal Treatment}

In addition to general oral health and visits to the general dentist, some studies have investigated the impact of fear and anxiety on preventing patients from seeking periodontal treatment. Desire for anesthesia can serve as an indicator of dental fear or anxiety. Using this premise, it is possible that individuals who desire sedation or general anesthesia experience the greatest negative perception toward periodontal surgery. In the surveyed population, preference for sedation was highest for periodontal surgery $(68.2 \%)$ compared to endodontic procedures (54.7\%) or extraction (46.5\%) [22].

Patients who have greater anxiety regarding a periodontal procedure ultimately experience a greater amount of pain compared to patients with low levels of anxiety [30]. With respect to dental implant surgery, anxiety was the strongest predictor of the amount of pain a patient would experience following surgery [30]. Similarly, another study found that patients with high anxiety prior to periodontal surgery experienced significantly more pain than those with low anxiety prior to periodontal surgery [31].

\section{Strategies for Reducing Dental Fear and Anxiety}

It is critical to remove as many of the barriers to dental treatment as possible so patients will seek required dental treatment. Receiving the appropriate dental treatment in a timely manner should help individuals to maintain their natural teeth or to receive dental implants so that they are able to eat a healthy and varied diet. As shown in Table 2, there are a variety of potential strategies that have been used to manage anxiety in the dental setting.

Patient concerns regarding dental visits differ greatly based on how they perceive the dentist and dental treatment. Some psychological aspects of dental treatment are the cause of a patient's 
nervousness. Previous reports have shown that patients report more anxiety toward dental treatment when they have previously had a painful dental experience [27,32]. For example, it was found that the severity of a previous negative experience influenced an individual with high dental anxiety to avoid dental treatment [27]. Negative experiences that were explored included pain, discomfort, gagging, fainting or feeling light-headed, embarrassment, and having a personal problem with the dentist. Among patients with high dental anxiety, those who had previously fainted or felt embarrassment because of dental treatment had a significantly greater chance of dental avoidance. For those with low dental anxiety, there was a significant relationship between a previous experience involving pain or fainting that they considered to be strongly negative and current dental avoidance [27]. It has also been reported that some patients feel anxious when there is a perceived loss of control in their treatment $[32,33]$. It is important in these situations that the dental professional is able to identify nervous patients and effectively communicate with them. This helps build trust between the patient and the dental professional. In turn, this can reduce anxiety and help the patient to feel more in control of their treatment [32]. Effective communication between the dental staff and the patient is essential for building trust in the relationship and putting the patient at ease. According to a study of a group of patients who had high dental anxiety but who managed to overcome their anxiety to seek regular dental care, a foundation of trust was key to making this possible. Communication was identified as a pillar in building the trust in this relationship [34].

Some aspects of dental treatment that trigger anxiety are physically uncomfortable experiences such as bad tastes, receiving the local anesthetic or having too much fluid in the mouth [31]. A study of negative experiences at consecutive surgeries showed there was a poor correlation between the specific experiences that were causing discomfort. It was hypothesized that this was because if a patient had an uncomfortable experience during the first surgery, then the patient was able to communicate this to the surgeon to avoid the negative experience in the following visit [31]. This is an encouraging finding, but again it emphasizes the need for effective communication between the patient and the clinician.

Creating an environment where a patient feels at ease can also help to reduce fear and anxiety. The reception area of dental offices is an opportunity to do this. To determine if aromatherapy could reduce anxiety in the reception area, patients were divided into four groups: a control with no intervention, a group who listened to music, a group exposed to the scent of orange, and a group exposed to the scent of lavender. Significant differences were found between the control and the orange scent group and between the control and the lavender scent group, but not between the control and the music group. Those in the aromatherapy groups reported less anxiety and a greater feeling of calmness [35]. While there was no benefit with the music intervention [35], there is some evidence to show that music therapy can help reduce anxiety, albeit not as effectively as other methods [36]. A brief relaxation technique, music therapy, and a control were compared on their efficacy for decreasing dental anxiety. Anxiety decreased in both the relaxation and music groups compared to the control, but anxiety in the relaxation group was also significantly lower than the music group. The reduction in anxiety in the relaxation group was the only one deemed clinically relevant [36].

Functional relaxation is an example of a behavioural therapy that has been explored to reduce anxiety; other examples include distraction using virtual reality and hypnosis. The virtual reality intervention included various relaxing natural environments such as a beach, a forest, or mountains that the patient could navigate. Patients experienced $5 \mathrm{~min}$ of treatment without the distraction then 5 min of treatment with the distraction. Self-evaluation and physiological measures supported the notion than distraction helps reduce anxiety in a dental setting [37]. To look at the effects of hypnosis, patients were assigned to either the treatment only group or treatment with hypnosis. Hypnosis was achieved by having patients listen to a recording. After treatment, patients from both groups were asked to rate their anxiety during treatment and after treatment. Those in the hypnosis group reported having lower levels of anxiety during treatment [38].

If a patient experiences greater levels of fear or anxiety, to the extent where communication and giving the patient a sense of control in the treatment is not sufficient to allow them to feel at ease, 
sedation or general anesthesia is a viable alternative [22]. A total of $31.1 \%$ of patients with high dental fear were 'definitely interested' in sedation, another $54.1 \%$ of those patients indicated they 'might be interested' depending on cost [22]. Interestingly, even some patients who reported low levels of dental fear were interested in sedation [22]. Another study examined the differences in dental anxiety between a group who had local anesthetic alone and a group who had local anesthetic combined with conscious sedation. Patients who had sedation plus local anesthetic reported less pain when they arrived before the surgery and recalled (1-month post-surgery) less pain and a lower level of predicted and recalled anxiety. It was suggested that if individuals recall less pain and anxiety following treatment, they are more likely to seek treatment in the future [39].

It is important for a clinician to identify patients who are anxious about treatment so the clinician is able to make them feel comfortable about the process. Encouraging dentally anxious patients to ask questions regarding their procedure might make them feel at ease, and thus more likely to seek the treatment they need to restore oral health and allow them to live a more healthful lifestyle overall. Clearly, finding effective strategies to eliminate or manage anxiety in a dental setting is a key to breaking the cycle shown in Figure 1. Further study is needed to develop such strategies.

Table 2. Strategies to manage anxiety in a dental setting.

\begin{tabular}{cl}
\hline Strategy & \multicolumn{1}{c}{ Evidence of Efficacy } \\
\hline Communication & $\begin{array}{l}\text { Effective communication between the dental staff and the patient is essential for } \\
\text { building trust in the relationship and putting the patient at ease [34]. }\end{array}$ \\
\hline Sedation or General Anesthesia & $\begin{array}{l}\text { Sedation can lower a patient's current state of pain and recalled pain. It can also } \\
\text { lower predicted and recalled anxiety [39]. }\end{array}$ \\
\hline Relaxation & $\begin{array}{l}\text { When patients were given instructions on functional relaxation and performed it } \\
\text { during their treatment, it significantly lowered their anxiety [36]. }\end{array}$ \\
\hline Distraction & $\begin{array}{l}\text { Virtual reality was tested to determine if distraction from the dental treatment } \\
\text { reduced anxiety. Patients' self-evaluation and physiological measures showed } \\
\text { that distraction helps reduce anxiety in a dental setting [37]. }\end{array}$ \\
\hline Hypnosis & $\begin{array}{l}\text { Patients who were hypnotized reported having lower levels of anxiety during } \\
\text { treatment than those who were not [38]. }\end{array}$ \\
\hline Aromatherapy & $\begin{array}{l}\text { Patients in the aromatherapy groups reported less anxiety and a greater feeling of } \\
\text { calmness [35]. }\end{array}$ \\
\hline
\end{tabular}

\section{Conclusions}

The literature demonstrates that oral health status affects food and nutrient intake. It can influence the perceived ease with which individuals eat different foods and there is some evidence that individuals with fewer teeth avoid foods that can be considered difficult to chew $[10,15]$. Individuals with fewer teeth have been found to have lower intake or status of some nutrients, such as vitamin C, compared to individuals with more teeth [11]. It can be concluded that fear is a reason patients avoid dental treatment and some evidence suggests that periodontal surgery might cause patients the greatest anxiety compared to other types of dental surgery [22,28]. This dental fear and anxiety can also lead to avoiding periodontal surgery. Current knowledge gaps include identifying how the nutritional status of patients with dental anxiety compares to patients who do not suffer from dental anxiety and whether restoring the dentition leads to more variety in food choice. It is reasonable that if the cycle can be interrupted through further understanding of this complex relationship, patients are likely to live more healthfully and the patient can be made less apprehensive about the dental experience.

Acknowledgments: Wendy E. Ward holds a Canada Research Chair in Bone and Muscle Development.

Author Contributions: Jennifer R. Beaudette wrote the first draft of this review and took the lead with revisions. Wendy E. Ward and Peter C. Fritz conceived the framework for the review. Peter C. Fritz and Philip J. Sullivan provided constructive guidance and feedback. Wendy E. Ward provided conceptual guidance throughout the writing of the review.

Conflicts of Interest: The authors declare no conflict of interest. 


\section{Abbreviations}

The following abbreviations are used in this manuscript:

$\begin{array}{ll}\text { CVD } & \text { Cardiovascular disease } \\ \text { DALY } & \text { Disability adjusted life year } \\ \text { HEI } & \text { Healthy Eating Index } \\ \text { IDAF-4C } & \text { Index of Dental Anxiety and Fear-4C } \\ \text { NHANES } & \text { National Health and Nutrition Examination Survey } \\ \text { POPs } & \text { Posterior occluding pairs }\end{array}$

\section{References}

1. Ryden, L.; Buhlin, K.; Ekstrand, E.; de Faire, U.; Gustafsson, A.; Holmer, J.; Kjellstrom, B.; Lindahl, B.; Norhammar, A.; Nygren, A.; et al. Periodontitis Increases the Risk of a First Myocardial Infarction: A Report From the PAROKRANK Study. Circulation 2016, 133, 576-583. [PubMed]

2. Artese, H.P.; Foz, A.M.; Rabelo Mde, S.; Gomes, G.H.; Orlandi, M.; Suvan, J.; D'Aiuto, F.; Romito, G.A. Periodontal therapy and systemic inflammation in type 2 diabetes mellitus: A meta-analysis. PLoS ONE 2015, 10, e0128344. [CrossRef] [PubMed]

3. Michaud, D.S.; Kelsey, K.T.; Papathanasiou, E.; Genco, C.A.; Giovannucci, E. Periodontal disease and risk of all cancers among male never smokers: An updated analysis of the Health Professionals Follow-up Study. Ann. Oncol. 2016, 27, 941-947. [CrossRef] [PubMed]

4. Zanella, S.M.; Pereira, S.S.; Barbisan, J.N.; Vieira, L.; Saba-Chujfi, E.; Haas, A.N.; Rosing, C.K. Periodontal disease, tooth loss and coronary heart disease assessed by coronary angiography: A cross-sectional observational study. J. Periodontal. Res. 2016, 51, 221-227. [CrossRef] [PubMed]

5. Ioannidou, E.; Swede, H.; Fares, G.; Himmelfarb, J. Tooth loss strongly associates with malnutrition in chronic kidney disease. J. Periodontol. 2014, 85, 899-907. [CrossRef] [PubMed]

6. Schleicher, R.L.; Carroll, M.D.; Ford, E.S.; Lacher, D.A. Serum vitamin C and the prevalence of vitamin C deficiency in the United States: 2003-2004 National Health and Nutrition Examination Survey (NHANES). Am. J. Clin. Nutr. 2009, 90, 1252-1263. [CrossRef]

7. Health Canada. Report on the Findings of the Oral Health Component of the Canadian Health Measures Survey 2007-2009; Government of Canada Publications: Ottawa, ON, Canada, 2010.

8. Marcenes, W.; Kassebaum, N.J.; Bernabe, E.; Flaxman, A.; Naghavi, M.; Lopez, A.; Murray, C.J. Global burden of oral conditions in 1990-2010: A systematic analysis. J. Dent. Res. 2013, 92, 592-597. [CrossRef] [PubMed]

9. Eke, P.I.; Dye, B.A.; Wei, L.; Slade, G.D.; Thornton-Evans, G.O.; Borgnakke, W.S.; Taylor, G.W.; Page, R.C.; Beck, J.D.; Genco, R.J. Update on Prevalence of Periodontitis in Adults in the United States: NHANES 2009 to 2012. J. Periodontol. 2015, 86, 611-622. [CrossRef] [PubMed]

10. Joshipura, K.J.; Willett, W.C.; Douglass, C.W. The impact of edentulousness on food and nutrient intake. J. Am. Dent. Assoc. 1996, 127, 459-467. [CrossRef] [PubMed]

11. Sahyoun, N.R.; Lin, C.L.; Krall, E. Nutritional status of the older adult is associated with dentition status. J. Am. Diet. Assoc. 2003, 103, 61-66. [CrossRef] [PubMed]

12. Nowjack-Raymer, R.E.; Sheiham, A. Numbers of natural teeth, diet, and nutritional status in US adults. J. Dent. Res. 2007, 86, 1171-1175. [CrossRef] [PubMed]

13. Ranta, K.; Tuominen, R.; Paunio, I.; Seppanen, R. Dental status and intake of food items among an adult Finnish population. Gerodontics 1988, 4, 32-35. [PubMed]

14. Faller, A.L.; Fialho, E. Polyphenol availability in fruits and vegetables consumed in Brazil. Rev. Saude. Publ. 2009, 43, 211-218. [CrossRef]

15. Sheiham, A.; Steele, J.G.; Marcenes, W.; Finch, S.; Walls, A.W. The impact of oral health on stated ability to eat certain foods; findings from the National Diet and Nutrition Survey of Older People in Great Britain. Gerodontology 1999, 16, 11-20. [CrossRef] [PubMed]

16. Leake, J.L. An index of chewing ability. J. Public Health Dent. 1990, 50, 262-267. [CrossRef] [PubMed]

17. O'Neil, C.E.; Nicklas, T.A.; Fulgoni, V.L. Consumption of apples is associated with a better diet quality and reduced risk of obesity in children: National Health and Nutrition Examination Survey (NHANES) 2003-2010. Nutr. J. 2015, 14. [CrossRef] [PubMed] 
18. Koutsos, A.; Tuohy, K.M.; Lovegrove, J.A. Apples and cardiovascular health-Is the gut microbiota a core consideration? Nutrients 2015, 7, 3959-3998. [CrossRef] [PubMed]

19. Loney, R. Point of Care: Do missing teeth need to be replaced or is a shortened dental arch acceptable? J. Can. Dent. Assoc. 2007, 73, 593-594.

20. Sheiham, A.; Steele, J.G.; Marcenes, W.; Lowe, C.; Finch, S.; Bates, C.J.; Prentice, A.; Walls, A.W. The relationship among dental status, nutrient intake, and nutritional status in older people. J. Dent. Res. 2001, 80, 408-413. [CrossRef] [PubMed]

21. Yuan, G.F.; Sun, B.; Yuan, J.; Wang, Q.M. Effects of different cooking methods on health-promoting compounds of broccoli. J. Zhejiang Univ. Sci. B 2009, 10, 580-588. [CrossRef] [PubMed]

22. Chanpong, B.; Haas, D.A.; Locker, D. Need and demand for sedation or general anesthesia in dentistry: A national survey of the Canadian population. Anesth. Prog. 2005, 52, 3-11. [CrossRef]

23. Armfield, J.M.; Spencer, A.J.; Stewart, J.F. Dental fear in Australia: Who's afraid of the dentist? Aust. Dent. J. 2006, 51, 78-85. [CrossRef] [PubMed]

24. Liddell, A.; Locker, D. Gender and age differences in attitudes to dental pain and dental control. Community Dent. Oral. Epidemiol. 1997, 25, 314-318. [CrossRef] [PubMed]

25. Eli, I.; Baht, R.; Kozlovsky, A.; Simon, H. Effect of gender on acute pain prediction and memory in periodontal surgery. Eur. J. Oral. Sci. 2000, 108, 99-103. [CrossRef] [PubMed]

26. Heft, M.W.; Meng, X.; Bradley, M.M.; Lang, P.J. Gender differences in reported dental fear and fear of dental pain. Community Dent. Oral. Epidemiol. 2007, 35, 421-428. [CrossRef] [PubMed]

27. Armfield, J.M.; Ketting, M. Predictors of dental avoidance among Australian adults with different levels of dental anxiety. Health Psychol. 2015, 34, 929-940. [CrossRef] [PubMed]

28. Quteish Taani, D.S. Dental anxiety and regularity of dental attendance in younger adults. J. Oral. Rehabil. 2002, 29, 604-608. [CrossRef] [PubMed]

29. De Jongh, A.; Schutjes, M.; Aartman, I.H. A test of Berggren's model of dental fear and anxiety. Eur. J. Oral. Sci. 2011, 119, 361-365. [CrossRef] [PubMed]

30. Eli, I.; Schwartz-Arad, D.; Baht, R.; Ben-Tuvim, H. Effect of anxiety on the experience of pain in implant insertion. Clin. Oral. Implant. Res. 2003, 14, 115-118. [CrossRef]

31. Fardal, O.; McCulloch, C.A. Impact of anxiety on pain perception associated with periodontal and implant surgery in a private practice. J. Periodontol. 2012, 83, 1079-1085. [CrossRef] [PubMed]

32. Bare, L.C.; Dundes, L. Strategies for combating dental anxiety. J. Dent. Educ. 2004, 68, 1172-1177. [PubMed]

33. Maggirias, J.; Locker, D. Psychological factors and perceptions of pain associated with dental treatment. Community Dent. Oral. Epidemiol. 2002, 30, 151-159. [CrossRef] [PubMed]

34. Bernson, J.M.; Hallberg, L.R.; Elfstrom, M.L.; Hakeberg, M. 'Making dental care possible: A mutual affair': A grounded theory relating to adult patients with dental fear and regular dental treatment. Eur. J. Oral. Sci. 2011, 119, 373-380. [CrossRef] [PubMed]

35. Lehrner, J.; Marwinski, G.; Lehr, S.; Johren, P.; Deecke, L. Ambient odors of orange and lavender reduce anxiety and improve mood in a dental office. Physiol. Behav. 2005, 86, 92-95. [CrossRef] [PubMed]

36. Lahmann, C.; Schoen, R.; Henningsen, P.; Ronel, J.; Muehlbacher, M.; Loew, T.; Tritt, K.; Nickel, M.; Doering, S. Brief relaxation versus music distraction in the treatment of dental anxiety: A randomized controlled clinical trial. J. Am. Dent. Assoc. 2008, 139, 317-324. [CrossRef] [PubMed]

37. Wiederhold, M.D.; Gao, K.; Wiederhold, B.K. Clinical use of virtual reality distraction system to reduce anxiety and pain in dental procedures. Cyberpsychol. Behav. Soc. Netw. 2014, 17, 359-365. [CrossRef] [PubMed]

38. Glaesmer, H.; Geupel, H.; Haak, R. A controlled trial on the effect of hypnosis on dental anxiety in tooth removal patients. Patient Educ. Couns. 2015, 98, 1112-1115. [CrossRef] [PubMed]

39. Wilson, T.D.; McNeil, D.W.; Kyle, B.N.; Weaver, B.D.; Graves, R.W. Effects of conscious sedation on patient recall of anxiety and pain after oral surgery. Oral. Surg. Oral. Med. Oral. Pathol. Oral. Radiol. 2014, 117, 277-282. [CrossRef]

(C) 2017 by the authors; licensee MDPI, Basel, Switzerland. This article is an open access article distributed under the terms and conditions of the Creative Commons Attribution (CC BY) license (http://creativecommons.org/licenses/by/4.0/). 